\title{
Établissement de références en dose absorbée dans l'eau par calorimétrie dans l'eau au LNE-LNHB pour les rayons $X$ de moyenne énergie
}

\section{Absorbed dose to water standards established by water calorimetry at the $\mathrm{LNE}-\mathrm{LNHB}$ for medium energy $X$-ray}

\author{
Benjamin RAPP, Nicolas PÉRICHON, Marc DENOZIÈRE, Josiane DAURES, Aimé OSTROWSKY \\ et Jean-Marc BORDY
}

CEA, LIST, Laboratoire national Henri Becquerel (LNE-LNHB), 91191 Gif-sur-Yvette Cedex, France, benjamin.rapp@ cea.fr.

\section{Résumé}

Actuellement, la dose absorbée dans l'eau pour les rayons $\mathrm{X}$ de moyenne énergie est déterminée à partir de références en termes de kerma dans l'air et par application de protocoles internationaux de dosimétrie. De nouvelles références en termes de dose absorbée dans l'eau viennent d'être établies pour ces faisceaux au LNE-LNHB. Pour cela un calorimètre spécifique a été développé, permettant de faire des mesures à faible profondeur dans l'eau dans les conditions de références requises par les protocoles internationaux de dosimétrie pour les rayons $\mathrm{X}$ de moyenne énergie. Ce nouveau calorimètre a été utilisé pour mesurer le débit de dose absorbée à $2 \mathrm{~cm}$ de profondeur dans l'eau, de six faisceaux de rayons $\mathrm{X}$ de référence de moyenne énergie de $80 \mathrm{kV}$ à $300 \mathrm{kV}$. L'incertitude type relative obtenue sur le débit de dose absorbée par calorimétrie dans l'eau est inférieure à $0,8 \%$, alors que celle obtenue par application des protocoles fondés sur le kerma dans l'air est d'environ $2,5 \%$.

MOTS CLÉS : CALORIMÉTRIE DANS L'EAU, DOSE ABSORBÉE, RAYONS X DE MOYENNE ÉNERGIE.

\footnotetext{
Abstract

Nowadays, the absorbed dose to water for kilovoltage $X$-ray beams is determined from standards in terms of air-kerma by application of international dosimetry protocols. New standards in terms of absorbed dose to water have just been established for these beams at the LNELNHB. A specific calorimeter was developed to do measurements at low depth in water, in order to fulfill the reference conditions required
}

by the international dosimetry protocols for medium-energy X-ray. This new calorimeter was used to measure the absorbed dose rate in water at a depth of $2 \mathrm{~cm}$ for six medium-energy $X$-ray reference beams with a tube potential from $80 \mathrm{kV}$ to $300 \mathrm{kV}$. The relative standard uncertainty obtained on the absorbed dose rate by water calorimetry is lower than $0.8 \%$, whereas the one given by application of protocols based on airkerma is around $2.5 \%$.

KEYWORDS: WATER CALORIMETRY, ABSORBED DOSE, MEDIUM ENERGY X RAYS.

\section{Introduction}

Les protocoles de dosimétrie de l'AIEA (Agence internationale de l'énergie atomique) TRS-277 [1], AAPM (The American Association of Physicists in Medicine) TG61 [2], IPEMB (Institution of Physics and Engineering in Medicine and Biology,) [3] et NCS-10 (Nederlandse Commissie voor Stralingsdosimetrie) [4] pour les rayons $\mathrm{X}$ de moyenne énergie sont largement utilisés pour calculer la dose absorbée dans l'eau à partir de chambres d'ionisation étalonnées en kerma dans l'air. Quand des références en termes de dose absorbée dans l'eau sont disponibles, le protocole de l'AIEA TRS398 [5] peut être utilisé. Les protocoles TG-61, IPEMB 
Tableau 1

Caractéristiques des faisceaux de rayons $\mathrm{X}$ de référence pour lesquels des mesures par calorimétrie dans l'eau ont été effectuées.

\begin{tabular}{|c|c|c|c|c|}
\hline Faisceau & $\begin{array}{c}\text { Potentiel } \\
\text { du tube } \\
(\mathrm{kV})\end{array}$ & $\begin{array}{c}\text { Filtration } \\
\text { additionnelle } \\
(\mathrm{mm})\end{array}$ & $\begin{array}{c}\mathrm{CDA} \\
(\mathrm{mm})\end{array}$ & $\begin{array}{c}\text { Energie } \\
\text { moyenne } \\
(\mathrm{keV})\end{array}$ \\
\hline RQR6 & 80 & $2,50 \mathrm{Al}$ & $3,01 \mathrm{Al}$ & 44 \\
\hline RQR9 & 120 & $2,50 \mathrm{Al}$ & $5,00 \mathrm{Al}$ & 56 \\
\hline RQR10 & 150 & $2,50 \mathrm{Al}$ & $6,57 \mathrm{Al}$ & 63 \\
\hline CCRI180 & 180 & $4,06 \mathrm{Al}+0,51 \mathrm{Cu}$ & $1,00 \mathrm{Cu}$ & 85 \\
\hline CCRI250 & 250 & $4,02 \mathrm{Al}+1,72 \mathrm{Cu}$ & $2,50 \mathrm{Cu}$ & 120 \\
\hline ISOH300 & 300 & $4,00 \mathrm{Al}+2,50 \mathrm{Cu}$ & $3,40 \mathrm{Cu}$ & 141 \\
\hline
\end{tabular}

et NCS-10 préconisent une profondeur de référence dans l'eau de $2 \mathrm{~cm}$, alors que le protocole TRS-277 recommande une profondeur de $5 \mathrm{~cm}$. Le protocole TRS-398 préconise quant à lui une profondeur de référence dans l'eau de $2 \mathrm{~g} \cdot \mathrm{cm}^{-2}$. Pour tous ces protocoles, le champ de référence est un champ de $(10 \times 10) \mathrm{cm}^{2}$ à une distance de $50 \mathrm{~cm}$ à $100 \mathrm{~cm}$ de la source de rayons X. Des laboratoires nationaux de dosimétrie comme le VSL (Van Swinden Laboratorium, Pays-Bas) [6] et le PTB (Physikalisch-Technische Bundesanstalt, Allemagne) [7] ont déjà établi des références par calorimétrie dans l'eau pour les rayons $\mathrm{X}$ de moyenne énergie, mais dans des conditions de référence différentes de celles recommandées par les protocoles, notamment pour la profondeur de mesure dans l'eau. Ceci est dû au fait que les calorimètres à eau utilisés ont d'abord été conçus pour effectuer des mesures avec des photons de haute énergie pour lesquels la profondeur de référence est de $5 \mathrm{~g} \cdot \mathrm{cm}^{-2}$ ou $10 \mathrm{~g} \cdot \mathrm{cm}^{-2}$. C'est pourquoi le LNE-LNHB a développé un à eau spécifique permettant de réaliser des mesures à faible profondeur dans l'eau (jusqu'à $0,5 \mathrm{~cm}$ par rapport à la surface du fantôme d'eau) dans les conditions recommandées par les protocoles internationaux de dosimétrie pour les rayons X de moyenne énergie [8]. Ainsi, une comparaison plus directe entre les mesures primaires de la dose absorbée dans l'eau, et le résultat de l'application des protocoles basés sur le kerma dans l'air est possible $[9,10]$.

Le calorimètre nouvellement construit a été utilisé pour mesurer le débit de dose absorbée dans l'eau pour six faisceaux de référence de rayons $\mathrm{X}$ de moyenne énergie entre $80 \mathrm{kV}$ et $300 \mathrm{kV}$.

\section{Faisceaux de référence de rayons $X$ de moyenne énergie}

\subsection{Choix des faisceaux de référence}

Parmi les nombreux faisceaux de rayons $\mathrm{X}$ de moyenne énergie existants, six ont été sélectionnés dans les normes et protocoles en vigueur afin de couvrir la plage d'intérêt (tension d'accélération comprise entre $80 \mathrm{kV}$ et $300 \mathrm{kV}$ ). Il s'agit des faisceaux IEC 61267 RQR6; IEC 61267 RQR9; IEC 61267 RQR10; CCRI180; CCRI250 et ISOH300 (tab. 1). Ce choix s'est fait également en fonction des

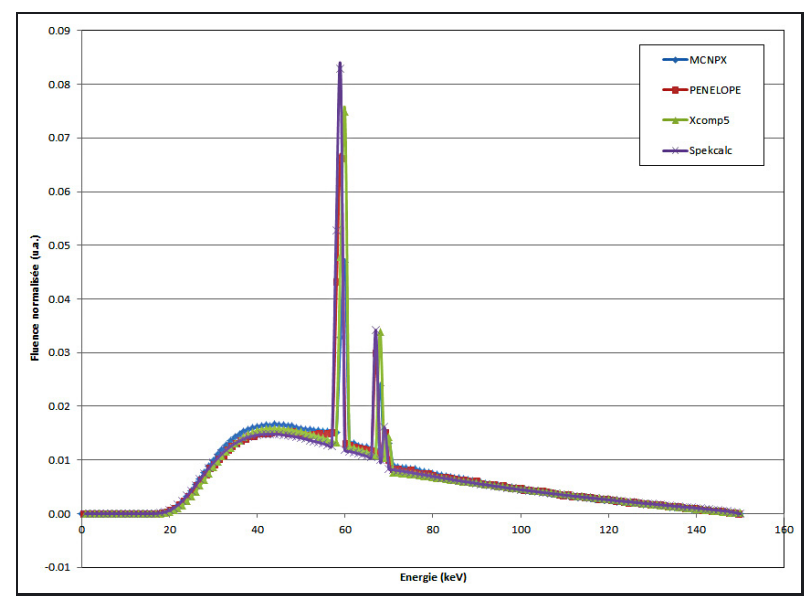

Fig. 1. - Représentation graphique des distributions en énergie de la fluence du faisceau RQR10 $(150 \mathrm{kV})$, calculées et issues de publications scientifiques.

performances du générateur de rayons $\mathrm{X}$ utilisé pour les mesures, parmi les faisceaux déjà caractérisés en kerma dans l'air au LNE-LNHB [11] et pour lesquels le débit de dose absorbée dans l'eau est assez élevé (> 0,3 Gy· $\mathrm{min}^{-1}$ ) pour réaliser des mesures par calorimétrie dans l'eau.

\subsection{Caractérisation des faisceaux de référence}

La chambre à parois d'air, instrument de référence du LNE-LNHB pour le kerma dans l'air des rayons X de moyenne énergie a été utilisée pour la mesure des couches de demi-atténuation (CDA), actuel indicateur de la qualité des faisceaux associé à la tension d'accélération.

La stabilité du générateur de rayons $X$ au cours du temps a également été vérifiée pour chacun des faisceaux de référence en effectuant des mesures périodiques sur plusieurs jours avec une chambre d'ionisation. Les résultats montrent qu'il n'est pas nécessaire de suivre le faisceau continuellement à l'aide d'une chambre moniteur (stabilité meilleure que $0,1 \%$ sur une semaine), et que ces variations aléatoires se trouvent contenues dans les incertitudes de mesure. La stabilité du générateur étant satisfaisante, il est donc possible d'étalonner des chambres d'ionisation de transfert en termes de kerma dans l'air et dose absorbée dans l'eau.

Pour chaque faisceau, les profils de «dose » dans l'air et dans l'eau et les rendements en profondeur dans l'eau ont été mesurés avec des chambres d'ionisation.

La distribution en énergie de la fluence des faisceaux a été déterminée par calcul. Ne disposant pas des données expérimentales, une modélisation du montage expérimental a été effectuée au moyen des codes Monte-Carlo MCNPX [12] et PENELOPE [13]. La comparaison des distributions en énergie calculées à celles issues de données publiées indique un bon accord (fig. 1). De plus, les bons résultats de la comparaison des profils et des rendements en profondeur dans l'eau simulés et mesurés 


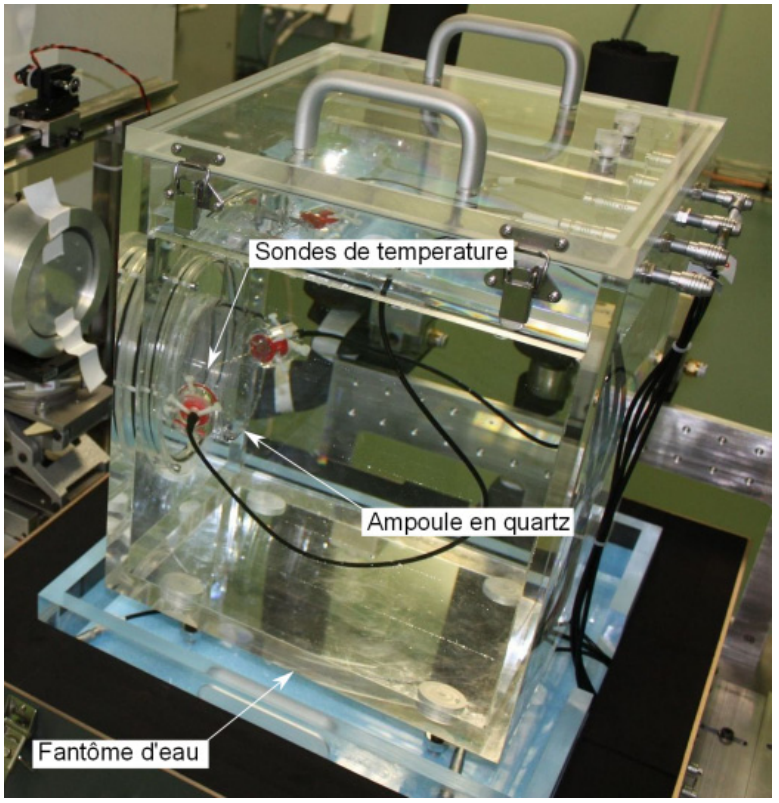

Fig. 2. - Vue de côté du calorimètre à eau permettant de réaliser des mesures à faible profondeur dans l'eau.

permettent d'être confiants quant à la bonne correspondance entre les spectres en énergie calculés et réels.

\section{Calorimètre à eau}

\subsection{Description générale}

Le nouveau calorimètre à eau du LNE-LNHB a été construit en tenant compte de l'expérience acquise lors de la conception et de l'utilisation du calorimètre à eau construit précédemment pour les faisceaux de photons de haute énergie $[14,15]$. Ainsi, il a été conçu pour opérer à $4{ }^{\circ} \mathrm{C}$, température à laquelle la masse volumique de l'eau est maximale, ceci afin de minimiser les courants de convection à l'intérieur du volume d'eau utilisé pour les mesures. L'intérieur du calorimètre consiste en un fantôme d'eau de radiothérapie de $(30 \times 30 \times$ 35) $\mathrm{cm}^{3}$ construit en PMMA (Polymethyl methacrylate) de $15 \mathrm{~mm}$ d'épaisseur, et rempli d'eau déminéralisée (fig. 2 et 3 ).

L'augmentation de température sous irradiation est mesurée par deux sondes de température, placées à l'intérieur d'une ampoule cylindrique en quartz remplie avec de l'eau ultrapure. Cette ampoule en quartz peut être insérée dans la face avant du fantôme d'eau (pour effectuer des mesures à faible profondeur dans l'eau) ou placée à n'importe quelle profondeur dans le fantôme d'eau. Pour être insérée dans la face avant du fantôme, l'ampoule est préalablement scellée dans un anneau en PMMA avec un joint silicone ; cet anneau est ensuite fixé au fantôme dans une ouverture circulaire prévue à cet effet. L'étanchéité entre les deux est assurée par un joint torique.

La température du fantôme d'eau est régulée à $4{ }^{\circ} \mathrm{C}$ par une circulation d'air froid à l'intérieur d'un espace d'épaisseur $20 \mathrm{~mm}$ situé entre le fantôme et l'isolation

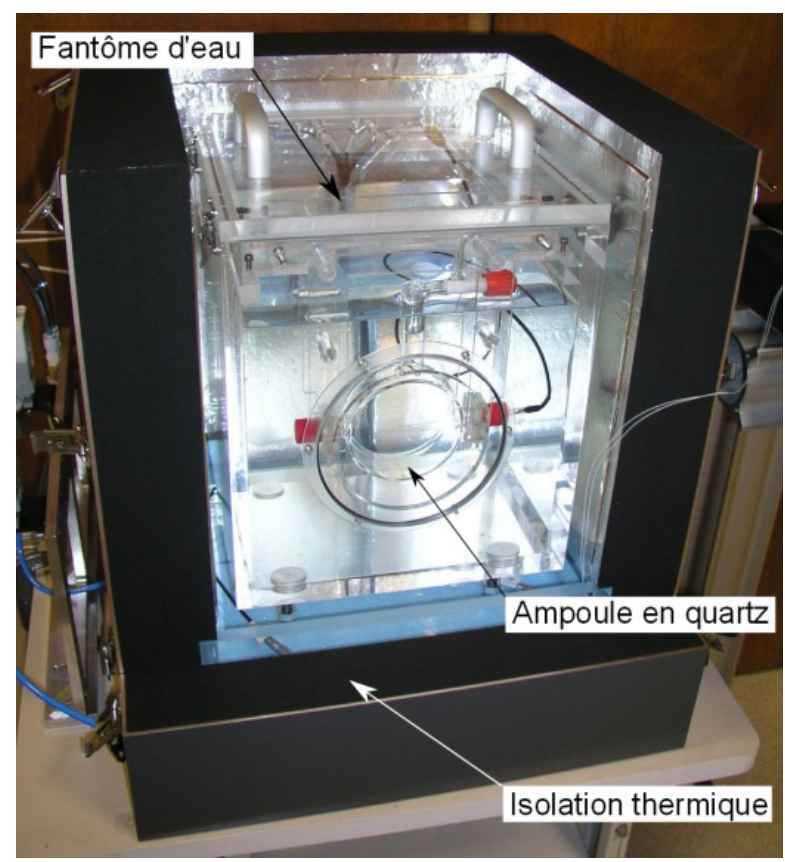

Fig. 3. - Vue de face du calorimètre à eau permettant de réaliser des mesures à faible profondeur dans l'eau.

thermique du calorimètre. L'isolation thermique du calorimètre est assurée par une couche épaisse $(80 \mathrm{~mm})$ de polystyrène expansé recouverte par $4 \mathrm{~mm}$ de PVC (polyvinyl chloride). Une fenêtre de $(12 \times 12) \mathrm{cm}^{2}$ est située dans l'axe du faisceau, l'enceinte thermique ayant à cet endroit une épaisseur réduite à $24 \mathrm{~mm}$ de polystyrène expansé (plus une feuille de Mylar de 0,1 mm).

\subsection{Système de régulation en température}

La circulation d'air froid à $4{ }^{\circ} \mathrm{C}$ environ est assurée par deux entrées, l'une placée sous la cuve d'eau et la seconde au niveau de la fenêtre d'entrée. Cet air froid est produit par des tubes Vortex (fig. 4), qui permettent de générer deux flux d'air à partir d'air comprimé, l'un plus froid et l'autre plus chaud que l'air initial. En réglant la pression de l'air d'alimentation, la température des flux d'air produits peut être modifiée. La relation entre la température de ces derniers et la pression n'est pas linéaire et dépend également du débit et de la température de l'air comprimé injecté. Pour réguler la température du flux d'air froid, un régulateur PID programmé sous LabVIEW est utilisé. Une thermistance placée à proximité de l'injection d'air froid dans le calorimètre sert de sonde de régulation. Sa résistance est mesurée directement par un voltmètre en mode ohmmètre et vient alimenter une boucle PID (proportionnelle intégrale dérivée) sous LabVIEW qui agit sur un régulateur de pression commandé par l'intermédiaire d'une carte source de tension programmable. Deux de ces systèmes sont nécessaires pour assurer la régulation et la circulation d'air froid de l'enceinte du calorimètre, l'un apportant de l'air à $3{ }^{\circ} \mathrm{C}$ sous la cuve et l'autre injectant de l'air à $4{ }^{\circ} \mathrm{C}$ au niveau de la fenêtre d'entrée. Avec ce système, la dérive thermique maximale mesurée dans le calorimètre, au 


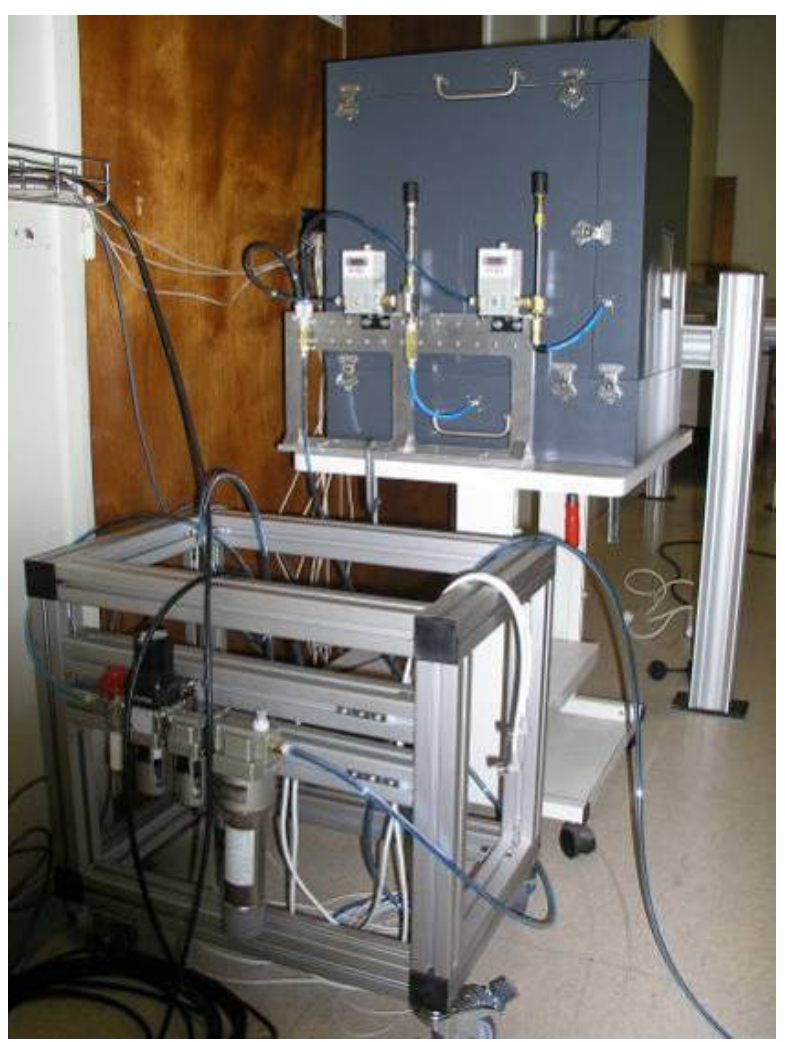

Fig. 4. - Système de refroidissement par circulation d'air utilisant des tubes Vortex. Au premier plan les différents filtres pour l'air comprimé du réseau, en amont des deux régulateurs de pression et des deux tubes Vortex au second plan devant l'enceinte du calorimètre (de couleur grise).

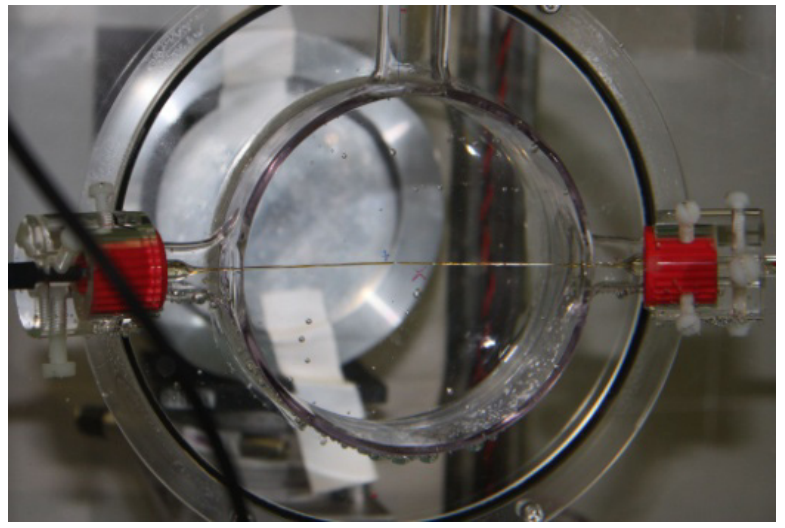

Fig. 5. - Ampoule scellée remplie d'eau ultrapure, encastrée dans la face avant du fantôme d'eau.

niveau de l'ampoule, est de $8 \mu \mathrm{K} \cdot \mathrm{min}^{-1}$. C'est une stabilité tout à fait satisfaisante, qui permet d'effectuer des mesures calorimétriques dans de très bonnes conditions.

\subsection{L'ampoule de confinement de l'eau ultrapure}

L'ampoule en quartz insérée dans la face avant de la cuve «évidée » mesure $10 \mathrm{~cm}$ de diamètre, $5 \mathrm{~cm}$ d'épaisseur et présente des surfaces planes de $0,8 \mathrm{~mm}$ d'épaisseur (fig. 5). Cette ampoule hermétiquement scellée

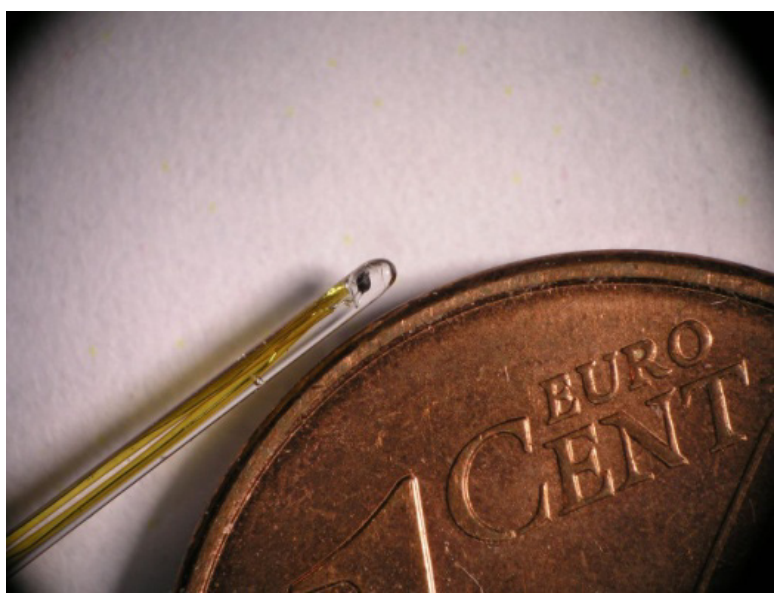

Fig. 6. - Extrémité d'une sonde de température. La thermistance de mesure (perle noire) est visible à l'extrémité du capillaire en quartz.

contient de l'eau ultrapure saturée en azote. La saturation de l'eau en azote permet d'obtenir un défaut de chaleur $h$ de l'eau pratiquement nul, c'est-à-dire que toute l'énergie déposée dans ce volume d'eau par le rayonnement est transformée en chaleur. Les mesures sont faites grâce à deux sondes de température (constituées chacune d'une thermistance insérée dans un tube capillaire en quartz), placées symétriquement au centre géométrique de l'ampoule (fig. 6). Le positionnement des sondes de température à l'intérieur de l'ampoule est réalisé au moyen d'un système optique composé d'une caméra et d'un objectif de microscope à fort grossissement montés sur une platine micrométrique. Afin de déterminer la profondeur de la sonde dans l'ampoule, la mise au point est réalisée successivement sur la paroi externe de l'ampoule et les thermistances à l'extrémité des sondes. A partir de la distance mesurée et en appliquant des corrections liées aux différences de parcours optique dans les milieux traversés (quartz, eau), la profondeur des sondes dans l'ampoule est déterminée avec une incertitude de $50 \mu \mathrm{m}$.

\section{Principe de mesure et facteurs de correction de la calorimétrie dans l'eau}

La dose absorbée dans l'eau par calorimétrie est obtenue à partir de l'équation suivante faisant intervenir différents facteurs correctifs :

$$
D_{\mathrm{w}}=C_{p} \Delta T(1-h)^{-1} k_{\mathrm{c}} k_{\mathrm{p}} k_{\rho} k_{\mathrm{prof}}
$$

avec, $D_{\mathrm{w}}$ la dose absorbée dans l'eau (en Gy), $C_{p}$ la capacité thermique massique de l'eau à pression constante (en $\mathrm{J} \cdot \mathrm{kg}^{-1} \cdot \mathrm{K}^{-1}$ ), $\Delta T$ la mesure de l'élévation de température sous irradiation (en K), $h$ le défaut de chaleur de l'eau, $k_{\mathrm{c}}$ le facteur de correction de la conduction thermique, $k_{\mathrm{p}}$ le facteur de correction des perturbations dosimétriques, $k_{\rho}$ le facteur de correction de la densité de l'eau, et $k_{\text {prof }}$ le facteur de correction de la profondeur des sondes. 


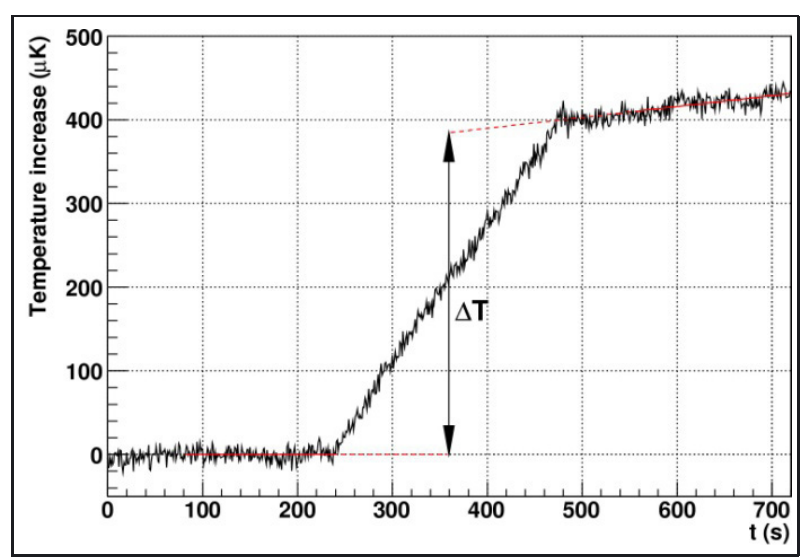

Fig. 7. - Exemple de mesure de l'élévation de température sous irradiation $(\Delta T)$ pour le faisceau ISOH300.

\subsection{Capacité thermique de l'eau à pression constante $\left(C_{p}\right)$}

La plupart des laboratoires nationaux de métrologie s'accordent pour prendre $4204,8 \mathrm{~J} \cdot \mathrm{kg}^{-1} \cdot \mathrm{K}^{-1}[16,17]$ comme valeur de la capacité thermique massique de l'eau à $4{ }^{\circ} \mathrm{C}$. L'incertitude sur les données expérimentales originales de la capacité thermique massique de l'eau est mal documentée, mais estimée entre $0,01 \%$ et $0,02 \%$. La valeur de l'incertitude de type B relative sur la capacité thermique massique de l'eau a donc été élargie à 0,10\%.

\subsection{Mesure de l'élévation de température sous irradiation $(\Delta T)$}

La mesure de l'élévation de température sous irradiation est effectuée au moyen des deux sondes de température positionnées dans l'ampoule à $2 \mathrm{~cm}$ de profondeur dans l'eau. Un exemple de mesure est donné en figure 7. Pour chaque faisceau, plusieurs séries de mesures ont été réalisées. Chaque série est composée de 3 irradiations successives de 4 min. L'incertitude sur la mesure de l'élévation de température est considérée comme l'incertitude statistique sur la moyenne des mesures. Celle-ci est comprise entre $0,23 \%$ et $0,40 \%$ en fonction du faisceau considéré (incertitude type relative de type A).

\subsection{Défaut de chaleur de l'eau $(h)$}

Le défaut de chaleur de l'eau est défini par la relation : $h=\frac{E_{\mathrm{a}}-E_{\mathrm{h}}}{E_{\mathrm{a}}}$,

avec $E_{\mathrm{a}}$ l'énergie absorbée par le milieu, c'est-à-dire l'énergie déposée par le rayonnement, et $E_{\mathrm{h}}$ l'énergie apparaissant sous forme de chaleur. Ce bilan n'est pas nul si l'énergie apportée par les rayonnements ionisants est incomplètement convertie en chaleur. En effet les rayonnements ionisants sont à l'origine du processus de radiolyse de l'eau qui initie un ensemble complexe de réactions chimiques dont le bilan thermique final peut être exothermique ( $h$ négatif) ou endothermique ( $h$ positif). La valeur du défaut de chaleur dépend fortement du contenu en oxygène dissous et en impuretés organiques de l'eau utilisée.

Le défaut de chaleur de l'eau ultrapure utilisée (saturée en azote de haute pureté) a été estimé par une simulation de la radiolyse de l'eau à $+0,1 \%$ pour les six faisceaux (endothermique). L'incertitude sur le défaut de chaleur $h$ a été estimée à $0,3 \%$ à partir d'une simulation numérique des réactions de radiolyse dans l'eau basée sur des mesures expérimentales du contenu en oxygène dissous et en impuretés organiques. Ainsi, en accord avec les autres laboratoires nationaux de métrologie [18-21], le choix a été fait de considérer un défaut de chaleur nul avec une incertitude associée de 0,3\%.

\subsection{Facteur de correction de la conduction thermique $\left(k_{\mathrm{c}}\right)$}

Le facteur de correction de la conduction thermique prend en compte les transferts de chaleur par conduction qui perturbent la mesure au niveau de la thermistance. Ceci provient de la grande différence de capacité thermique massique entre l'eau et le quartz constituant l'ampoule et les sondes de température.

Une modélisation de la géométrie du calorimètre a été réalisée à l'aide du code Monte-Carlo MCNPX, pour les six faisceaux, afin d'obtenir le dépôt d'énergie dans le calorimètre. Le calcul du dépôt d'énergie obtenu avec MCNPX sert ensuite de source de chaleur pour le calcul des transferts thermiques avec le logiciel de calcul par éléments finis COMSOL [22]. Pour des raisons de taille de maillage des structures utilisées dans le calcul par éléments finis, l'ampoule et la sonde sont modélisées séparément.

En comparant l'élévation de la température obtenue par le calcul par éléments finis avec l'élévation de la température attendue en l'absence de transferts thermiques, on en déduit les facteurs de correction de la conduction thermique (de la sonde et de l'ampoule) à appliquer à chaque irradiation. Les résultats des facteurs de correction de conduction thermique globaux (sonde et l'ampoule combinés) sont compris entre 1,005 6 et 1,040 6 selon le faisceau. L'incertitude type relative totale associée à ce facteur est comprise entre $0,13 \%$ et $0,44 \%$ en fonction du faisceau considéré. Cette incertitude est estimée en faisant varier les valeurs des paramètres du calcul aux éléments finis (dépôt d'énergie, taille du maillage, définition des matériaux).

\subsection{Facteur de correction des perturbations dosimétriques $\left(k_{\mathrm{p}}\right)$}

Les perturbations dosimétriques des mesures calorimétriques ont pour origine les matériaux autres que l'eau se trouvant sur le trajet du faisceau ou dans un environnement proche du point de mesure. Deux structures majeures d'influence ont été identifiées : l'enceinte thermique et l'ampoule en quartz. Les deux facteurs de correction relatifs à chacune de ces perturbations ont été 
déterminés de manière indépendante par mesure et par calcul Monte-Carlo.

Le facteur de correction des perturbations induites par l'enceinte thermique a été déterminé grâce à des mesures ionométriques effectuées avec une chambre d'ionisation. Deux séries de mesures, une sans et une avec enceinte thermique, ont été réalisées pour les six faisceaux. Le facteur de correction $k_{\mathrm{p}}$ de l'enceinte, qui est le rapport des deux mesures ionométrique, est compris entre 1,012 et 1,051 selon le faisceau. L'incertitude relative associée à la valeur du facteur est comprise entre $0,13 \%$ et $0,24 \%$. Il s'agit de l'incertitude type associée au rapport de deux mesures ionométriques. Le facteur de correction des perturbations dosimétriques induites par l'ampoule en quartz a été déterminé par simulations Monte-Carlo. Il est compris entre 1,002 et 1,013 selon le faisceau, avec une incertitude type relative de $0,11 \%$.

\subsection{Facteur de correction de la profondeur des sondes dans l'eau $\left(k_{\text {prof }}\right)$}

L'assemblage des sondes ne rend pas possible un positionnement exact à une profondeur de $2 \mathrm{~cm}$ dans l'eau. De plus, à cause des dimensions de l'enceinte thermique du calorimètre et du blindage du tube à rayons $\mathrm{X}$, la distance source-point de mesure pour les mesures calorimétriques est prise comme distance de référence. Lors de la comparaison ultérieure des valeurs de débits de dose absorbée, les séries de valeurs issues de l'application des protocoles internationaux sont ramenées à cette distance de référence. Ce facteur de correction est compris entre 1,000 7 et 1,001 7 selon le faisceau, avec une incertitude type relative comprise entre $0,04 \%$ à $0,22 \%$.

\section{7. Étalonnage des sondes}

Avant d'être utilisées dans le calorimètre, les sondes nécessitent un étalonnage en température. Pour cela, elles sont placées dans un bain thermostaté en même temps qu'une sonde de température de référence (Pt-100) étalonnée au LNE-LCM. L'incertitude type relative sur la mesure de l'élévation de température est de $0,1 \%$.

\subsection{Positionnement des sondes et du calorimètre}

L'incertitude type relative sur le positionnement des sondes de température et du calorimètre à eau est comprise entre $0,06 \%$ et $0,11 \%$.

\subsection{Temps d'irradiation}

L'incertitude type relative sur la mesure du temps d'irradiation, nécessaire au calcul du débit de dose, est de $0,03 \%$.

\subsection{Bilan d'incertitude}

Après avoir été validé dans le faisceau de ${ }^{60} \mathrm{Co}$ de référence du laboratoire, le calorimètre a été utilisé pour des
Tableau 2

Bilan d'incertitude sur le débit de dose absorbée dans l'eau mesuré par calorimétrie dans l'eau pour le faisceau CCRI180.

\begin{tabular}{|c|c|c|c|}
\hline \multirow{2}{*}{ Composantes } & \multirow{2}{*}{ Valeur } & \multicolumn{2}{|c|}{ Incertitude relative } \\
\hline & & Type A & Type B \\
\hline$C_{p}\left(\mathrm{~J} \cdot \mathrm{kg}^{-1} \cdot \mathrm{K}^{-1}\right)$ & 4204,80 & & $0,10 \%$ \\
\hline$\Delta T(\mu \mathrm{K})$ & 386,84 & $0,29 \%$ & \\
\hline$h$ & 0 & & $0,30 \%$ \\
\hline \multirow{3}{*}{$k_{\mathrm{c}}(1)$} & 1,0144 & & \multirow{3}{*}{$0,24 \%$} \\
\hline & 1,0107 & & \\
\hline & 1,0099 & & \\
\hline$k_{\mathrm{p}}$ & 1,0257 & & $0,18 \%$ \\
\hline$k_{\rho}$ & 1,0002 & & $0,03 \%$ \\
\hline$k_{\text {prof }}$ & 1,0008 & & $0,12 \%$ \\
\hline Étalonnage des sondes & - & & $0,10 \%$ \\
\hline $\begin{array}{c}\text { Positionnement des } \\
\text { sondes } \\
\end{array}$ & - & & $0,07 \%$ \\
\hline Temps d'irradiation & - & $0,03 \%$ & \\
\hline $\begin{array}{c}\text { Somme quadratique } \\
\text { des incertitudes }\end{array}$ & & $0,29 \%$ & $0,47 \%$ \\
\hline$D_{\mathrm{w}}\left(\mathrm{Gy} \cdot \mathrm{min}^{-1}\right)$ & 0,4244 & \multicolumn{2}{|c|}{$0,55 \%$} \\
\hline
\end{tabular}

(1) Les trois valeurs calculées du facteur de correction, servent à corriger séparément les trois paliers d'irradiations consécutifs de chaque série d'irradiation.

mesures dans les six faisceaux de rayons $\mathrm{X}$ de moyenne énergie sélectionnés. Les mesures calorimétriques ont été réalisées directement dans les conditions de référence des protocoles à $2 \mathrm{~cm}$ de profondeur dans l'eau et dans un champ carré de $(10 \times 10) \mathrm{cm}^{2}$ dans le plan de référence. L'incertitude type relative associée à la valeur du débit de dose absorbée dans l'eau ainsi obtenu est comprise entre $0,49 \%$ et $0,72 \%$ en fonction du faisceau utilisé. Un exemple de bilan d'incertitude sur le débit de dose absorbée dans l'eau mesuré par calorimétrie dans l'eau pour le faisceau CCRI180 est donné dans le tableau 2.

\section{Détermination de la dose absorbée dans l'eau à partir de références en termes de kerma dans l'air}

La dose absorbée dans l'eau est actuellement déterminée par application de protocoles de dosimétrie fondés sur l'utilisation d'un coefficient d'étalonnage en termes de kerma dans l'air. Dans le cadre de cette étude deux chambres d'ionisation ont été utilisées afin d'appliquer ces protocoles : une NE2571 et une PTW31010. Le coefficient d'étalonnage de ces chambres d'ionisation en termes de kerma dans l'air a été déterminé pour les six faisceaux retenus. Ensuite les coefficients d'étalonnage de ces chambres d'ionisation en dose absorbée dans l'eau ont étés mesurés dans les conditions de référence des protocoles, à $2 \mathrm{~cm}$ de profondeur dans l'eau. Quatre protocoles de dosimétrie ont alors été appliqués : AIEA TRS-277, AAPM TG-61, IPEMB, NCS-10. Les facteurs de correction nécessaires mais non disponibles dans les 


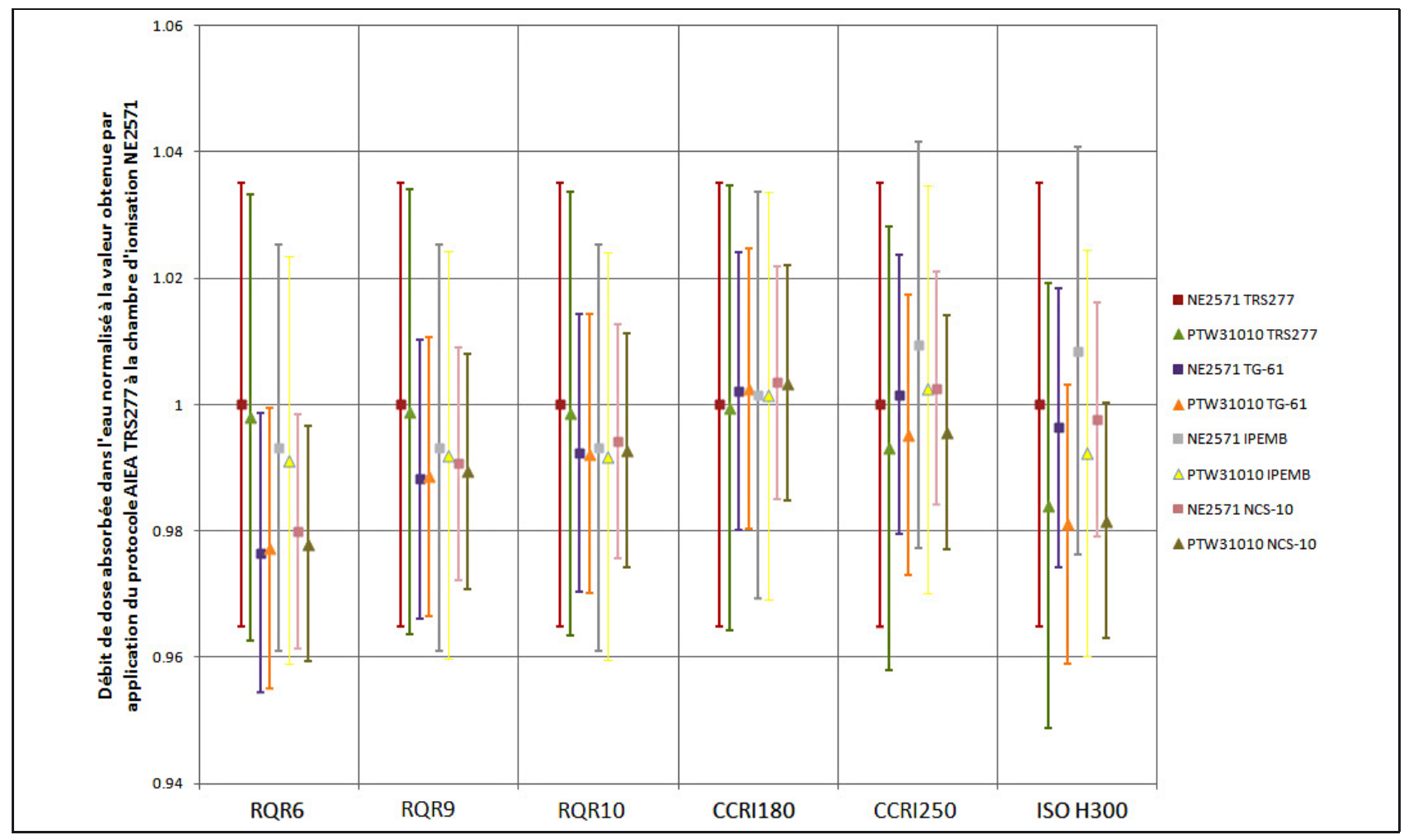

Fig. 8. - Comparaison des valeurs de débit de dose absorbée à $2 \mathrm{~cm}$ de profondeur dans l'eau par application de différents protocoles pour les chambres d'ionisation NE2571 et PTW31010 dans les six faisceaux d'intérêt.

protocoles ont été déterminés par mesures et/ou simulations Monte-Carlo. Les résultats issus de l'application des protocoles pour les deux chambres d'ionisation sont compatibles aux incertitudes près des méthodes quel que soit le faisceau (fig. 8).

De manière générale, l'application des protocoles nécessite l'utilisation de facteurs de correction parfois mal documentés et conduisant ainsi à une incertitude type totale élevée de la dose absorbée à $2 \mathrm{~cm}$ de profondeur dans l'eau, comprise entre $1,9 \%$ et 3,6\%, l'incertitude dépendant de la chambre d'ionisation, du faisceau et du protocole considéré.

\section{Comparaison des valeurs de débit de dose absorbée dans l'eau déterminées par application des protocoles et par calorimétrie}

Les valeurs de débit de dose absorbée dans l'eau obtenues par calorimétrie apparaissent en accord avec celles issues de l'application des différents protocoles pour les deux chambres d'ionisation (fig. 9). Les résultats sont tous compatibles aux incertitudes près des méthodes. Cependant, les incertitudes type associées à l'application des protocoles sont élevées (comprises entre 1,9\% et $3,6 \%$ ) en comparaison de celle obtenues par calorimétrie dans l'eau (comprises entre $0,49 \%$ et $0,72 \%$ ). Une amélioration significative des incertitudes est ainsi obtenue par application de la méthode calorimétrique par rapport à l'application des protocoles fondés sur le kerma dans l'air.

\section{Conclusion}

Des références directement en termes de dose absorbée dans l'eau, dans les conditions de référence des protocoles, ont été établies au LNE-LNHB par la méthode de la calorimétrie dans l'eau. La calorimétrie est la mesure de la dose absorbée à partir de l'élévation de température. Un calorimètre à eau spécifique a été conçu et réalisé afin d'effectuer des mesures à $2 \mathrm{~cm}$ de profondeur : condition de référence définie par le protocole AIEA TRS-398. L'incertitude type obtenue, sur le débit de dose absorbée mesuré par calorimétrie dans l'eau, est inférieure à $0,8 \%$.

Les références dosimétriques pour les rayons $\mathrm{X}$ de moyenne énergie en radiothérapie sont actuellement établies, le plus souvent, en termes de kerma dans l'air. La dose absorbée dans l'eau, grandeur d'intérêt pour la radiothérapie, est déduite de ces valeurs par transfert conformément aux protocoles internationaux avec des incertitudes comprises entre $1,9 \%$ et 3,6\%. Les débits de dose absorbée dans l'eau déterminés par calorimétrie ont été comparés aux valeurs issues de l'application des protocoles fondés sur le kerma dans l'air. Un écart maximum inférieur à $2 \%$ a été trouvé par rapport à la calorimétrie dans l'eau. L'incertitude type associée aux valeurs calorimétriques étant inférieure à $0,8 \%$ et celle associée aux valeurs issues des protocoles étant de l'ordre de 3,0\%, les résultats sont compatibles aux incertitudes des méthodes près. Grâce à ces nouvelles références, la détermination de la dose absorbée dans l'eau dans ce type de faisceau pourra désormais être réalisée en appliquant le protocole AIEA TRS-398, conduisant ainsi à une forte 


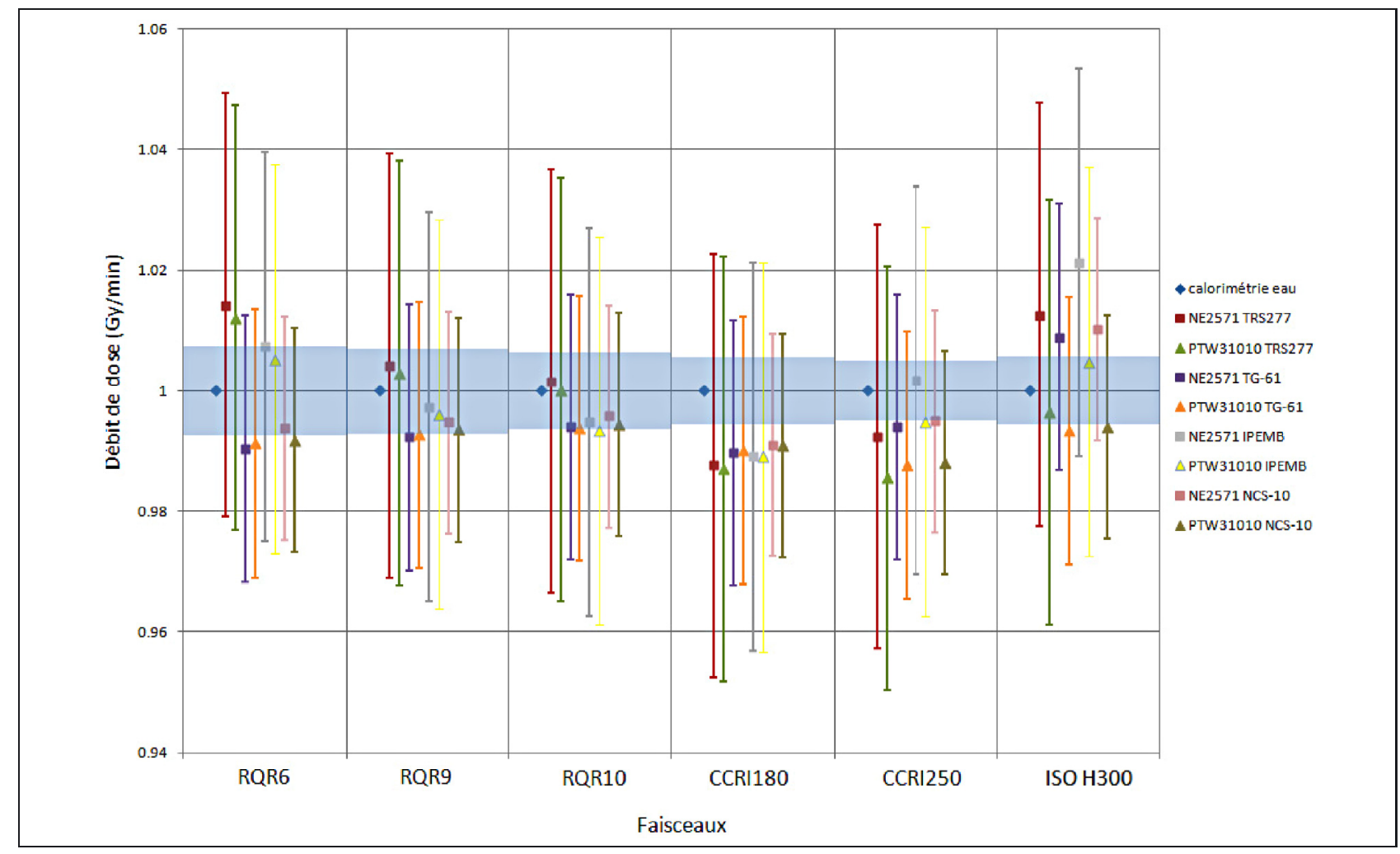

Fig. 9. - Comparaison des débits de dose absorbée dans l'eau déterminés par application des protocoles aux deux chambres d'ionisation (NE2571 et PTW31010) et ceux issus des mesures calorimétriques dans l'eau normalisés à la valeur issue des mesures calorimétriques dans l'eau; résultats pour les six faisceaux d'intérêt.

réduction des incertitudes (facteur 3 par rapport au protocole AIEA TRS-277). Actuellement, aucun autre laboratoire de dosimétrie ne possède un tel instrument permettant l'établissement direct de ces références dans les conditions recommandées par les protocoles. Une comparaison internationale de ces références est prévue dans le cadre du projet européen de métrologie EMRP HLT09.

\section{Références}

[1] "Absorbed dose determination in photon and electron beams", Technical Reports Series, 277, IAEA, 1997, Vienne.

[2] MA C.-M.(Chair), COFFEY C.W., DEWERD L.A., LiU C., NATH R., SELTZER S.M. et SEUNTJENS J.P., “AAPM protocol for 40-300 kV X-ray beam dosimetry in radiotherapy and radiobiology", Med. Phys., 28, 6, 2001868-93.

[3] Klevenhagen S.C. (Chair), Aukett R.J., Harrison R.M., MORETTI C., NAHUM A.E. et Rosser K.E., "The IPEMB code of practice for the determination of absorbed dose for X-rays below $300 \mathrm{kV}$ generating potential (0.035 mm Al-4 mm Cu HVL; 10-300 kV generating potential)", Phys. Med. Biol., 41, 12, 1996, 2605.

[4] Grimbergen T.W.M., AALBERS A.H.L., MiJNHEER B.J., SEUNTJENS J., THIERENS H., VAN DAM J., WITTK ÄMPER F.W. et ZoETELIEF J., "Dosimetry of low and medium energy X-rays - a code of practice for use in radiotherapy and radiobiology", NCS Report 10, Delft: Nederlandse Commissie voor Stralingsdosimetrie, 1997.

[5] "Absorbed dose determination in external beam radiotherapy”, Technical Reports Series, 398, IAEA 2000, Vienne.
[6] DE Prez L.A. et DE Pooter J.A., "The new NMi orthovolt X-rays absorbed dose to water primary standard based on water calorimetry", Phys. Med. Biol., 53, 13, 2008, 3531-3542.

[7] Krauss A., BÜERMANN L., Kramer H.-M. et SElbach H.-J., "Calorimetric determination of the absorbed dose to water for medium-energy X-rays with generating voltages from 70 to 280 kV", Phys. Med. Biol., 2012, 57, 19, 6245.

[8] Rapp B., Perichon N., Denoziere M., Daures J., OSTROWSKY A. et BORDY J.-M., "The LNE-LNHB water calorimeter for primary measurement of absorbed dose at low depth in water: application to medium energy X-rays”, Phys. Med. Biol., 58, 9, 2013, 2769.

[9] Perichon N., RapP B., Denoziere M., Daures J., OSTROWsKY A. et Bordy J.-M., "Comparison between absorbed dose to water standards established by water calorimetry at the LNE-LNHB and by application of international air-kerma based protocols for kilovoltage medium energy X-rays", Phys. Med. Biol., 58, 9, 2013, 2787.

[10] Périchon N, «Établissement des références nationales, en termes de dose absorbée, par calorimétrie dans l'eau, pour les faisceaux de rayons $\mathrm{X}$ de moyenne énergie, applicables en radiothérapie », Thèse de doctorat de sciences, Université Paris Sud, 10 sept. 2012.

[11] Burns D.T., Kessler C., Denoziere M. et Ksouri W., "Key comparison BIPM.RI(I)-K3 of the air-kerma standards of the LNE-LNHB, France and the BIPM in medium-energy X-rays", Metrologia, 45, Techn. Suppl., 2008, 06004. 
[12] "MCNPX Monte Carlo N-Particle Transport Code System for Multiparticle and High Energy Applications", http:// monpx.lanl.gov/.

[13] Salvat F., Fernandez-Varea J.-M., Acosta E. et Sempau J., "PENELOPE, A code system for Monte Carlo simulation of electron and photon transport", Proceedings of a Workshop/Training course, OECD/NEA, 5-7 novembre http://www.oecdnea.org/tools/abstract/detail/nea-1525/. la dosimétrie au LNE-LNHB », Rev. Fr Métrologie, 24, 2010, 3-8.

[15] RAPP B., "The LNE-LNHB water calorimeter: measurements in a ${ }^{60}$ Co beam IDOS", Int. Symp. On Standards, Applications and Quality Assurance in Medical Radiation Dosimetry, IAEA Proceedings Series STI/PUB/1514, Vienne, 9-12 nov. 2010, 1, 2011, 67. 2001, NEA/NSC/DOC(2001)19, ISBN:92-64-18475-9,

[14] RAPP B., "Développement d'un calorimètre à eau pour

[16] ZABRANSKY M., RUZICKA V.Jr, MAJER V. et DOMALSKI E.S., "Heat Capacity of Liquids: Volume I. Critical Review and Recommended Values", Monograph No. 6, J. Phys. Chem. Ref. Data, 1996.

[17] Osborne N.S., STIMSON H.F. et GinNings D.C., "Measurements of heat capacity and heat of vaporization of water in the range 0 to $100{ }^{\circ} \mathrm{C}^{\prime}$, J. Res. Natl Bur. Stand., 23, 1939, 197-260.

[18] SeuntJEns J. et DuANe S., "Photon absorbed dose standards", Metrologia, 46, 2, 2009, S39-S58.

[19] KLASSEN N.V. et Ross C.K., Water calorimetry: the heat defect, J. Res. Natl Inst. Stand. Technol., 102, 1997, 63-71.

[20] KLASSEN N.V. et Ross C.K., "Water calorimetry: a correction to the heat defect calculations", J. Res. Natl. Inst. Stand. Technol., 107, 2002, 171-8.

[21] Medin J., Ross C.K., StUCKi G., KLASSEN N.V. et SEUNTJENS J.P., "Commissioning of an NRC-type sealed water calorimeter at METAS using 60Co $\gamma$-rays", Phys. Med. Biol., 49, 17, 2004, 4073.

Article reçu le 11 septembre 2013, version révisée reçue le 15 septembre 2014. [22] COMSOL 4.2 Multiphysics, http://www.comsol.com/. 